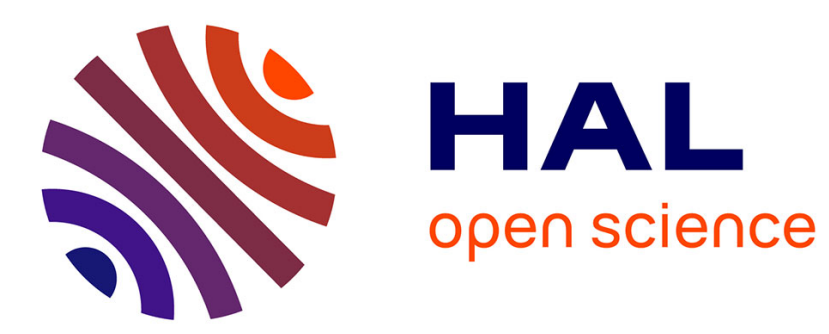

\title{
Robust fractional order differentiators using generalized modulating functions method
}

\author{
Da-Yan Liu, Taous-Meriem Laleg-Kirati
}

\section{To cite this version:}

Da-Yan Liu, Taous-Meriem Laleg-Kirati. Robust fractional order differentiators using generalized modulating functions method. Signal Processing, 2014. hal-00923748v3

\section{HAL Id: hal-00923748 \\ https://hal.inria.fr/hal-00923748v3}

Submitted on 5 May 2014

HAL is a multi-disciplinary open access archive for the deposit and dissemination of scientific research documents, whether they are published or not. The documents may come from teaching and research institutions in France or abroad, or from public or private research centers.
L'archive ouverte pluridisciplinaire HAL, est destinée au dépôt et à la diffusion de documents scientifiques de niveau recherche, publiés ou non, émanant des établissements d'enseignement et de recherche français ou étrangers, des laboratoires publics ou privés. 


\title{
Robust fractional order differentiators using generalized modulating functions method
}

\author{
Da-Yan Liu ${ }^{\mathrm{a}, \mathrm{b}}$, Taous-Meriem Laleg-Kirati ${ }^{\mathrm{b}}$ \\ ${ }^{a}$ INSA Centre Val de Loire, Université d'Orléans, PRISME EA 4229, 18022 Bourges, France \\ ${ }^{b}$ CEMSE Division, King Abdullah University of Science and Technology (KAUST), 23955-6900 Thuwal, KSA
}

\begin{abstract}
This paper aims at designing a fractional order differentiator for a class of signals satisfying a linear differential equation with unknown parameters. A generalized modulating functions method is proposed first to estimate the unknown parameters, then to derive accurate integral formulae for the left-sided Riemann-Liouville fractional derivatives of the studied signal. Unlike the improper integral in the definition of the left-sided Riemann-Liouville fractional derivative, the integrals in the proposed formulae can be proper and be considered as a low-pass filter by choosing appropriate modulating functions. Hence, digital fractional order differentiators applicable for on-line applications are deduced using a numerical integration method in discrete noisy case. Moreover, some error analysis are given for noise error contributions due to a class of stochastic processes. Finally, numerical examples are given to show the accuracy and robustness of the proposed fractional order differentiators.
\end{abstract}

Keywords: Fractional order differentiator, Riemann-Liouville derivative, Generalized modulating functions method, Noise.

\section{Introduction}

Fractional calculus was introduced in many fields of science and engineering long time ago. It was first developed by mathematicians in the middle of the ninetieth century [1]. During the past decades, fractional calculus has gained great interest in several applications [2, 3, 4]. For instance, fractional derivatives can improve the performances and robustness properties in control design (see, e.g. $[5,6,7,8]$ ) and in signal processing applications (see, e.g. [9, 10, 11, 12]). The fractional order numerical differentiation is concerned with the estimation of the fractional order derivatives of an unknown signal from its discrete noisy observed data. As in the integer order case, this problem is an ill-posed problem in the sense that a small noise can lead to a large error in approximated derivatives. In order to overcome this problem, various robust fractional order differentiators have been proposed in the frequency domain (see, e.g. [13, 14]) and in the time

Email addresses: dayan.liu@insa-cvl.fr (Da-Yan Liu), taousmeriem.laleg@kaust.edu.sa (Taous-Meriem Laleg-Kirati) 
domain, such as digital fractional order Savitzky-Golay differentiator [15], fractional order Jacobi differentiator [16], and B-Spline functions-based fractional order differentiator [17]. The main idea of the latter fractional order differentiators designed in the time domain is to use a polynomial to approximate the unknown signal whose fractional order derivatives are estimated by differentiating the polynomial. If we consider the used polynomial as the truncated Taylor series expansion of the unknown signal, then these fractional order differentiators contain an estimation error due to the truncated term in the Taylor series expansion. When estimating the fractional order derivatives of an unknown signal, even in noise free case, this kind of truncation errors can produce large errors near the boundaries of the interval where the fractional order derivatives are estimated $[16,17]$.

Existing fractional order differentiators are usually extensions of integer order differentiators $[18,19,20]$. When estimating the derivatives of an unknown signal, if the differentiators do not depend on any model which gives the unknown signal, then we call them model-free differentiators. In order to avoid truncation errors, integer order model-based differentiators have been proposed using a recent algebraic parametric method to estimate the state variables of an input-output linear system $[21,22,23]$. The successive integer order derivatives of the output were accurately estimated from its noisy observation without any truncation error. The idea of this algebraic parametric method is to apply the Laplace transform to the linear differential equation which defines the studied linear system. By applying some algebraic operations (such as differentiations and multiplications) in the Laplace operational domain, undesired terms in the obtained equation are eliminated. When returning into the time domain, the integer order derivatives of the output are exactly given by integral formulae involving the output and a combination of the weight functions of Jacobi orthogonal polynomials. Then, integer order differentiators are deduced by taking the noisy observation of the output in the obtained integral formulae. It has been shown in $[24,25]$ that thanks to the integral formulae these differentiators exhibit good robustness properties with respect to corrupting noises even if the statistical properties of the noises are unknown. Recall that the algebraic parametric method was introduced by Fliess and Sira-Ramírez for linear identification [26], and has been extended to many applications in noisy environment, such as design of integer order model-free differentiators (see, e.g. $[27,28,29,30,31]$ ) and parameter estimation (see, e.g. $[32,33,34,35,36,37])$. Very recently, it has been applied for fractional order model-free differentiators [38] and for fractional order systems identification [39]. However, the algebraic parametric method has not been applied for fractional order model-based differentiators.

Modulating functions method introduced by Shinbrot [40] is very similar to the algebraic parametric method. This method has been widely used for linear and non-linear identification of continuous-time systems (see, e.g. [41, 42, 43]), and parameter estimation of noisy sinusoidal signals (see, e.g. $[44,36])$. The idea of this method is to multiply a class of modulating functions to a linear differential equation of the analyzed signal. Then, an integration over a finite interval is 
taken to the obtained equation. Application of integration by parts allows to remove the derivative operations from the analyzed signal to the multiplied modulating functions, and the undesired boundary values are eliminated thanks to the properties of modulating functions. Finally, estimators are obtained by solving a linear system of algebraic equations and given by integral formulae involving the noisy observation of the analyzed signal. Since the weight functions of Jacobi orthogonal polynomials obtained in integral formulae by the algebraic parametric method are also a class of modulating functions, the modulating functions method can be considered as a generalization of the algebraic parametric method in some cases (see, e.g. [36]). It has similar advantages to the algebraic parametric method, especially the robustness properties with respect to corrupting noises. Moreover, inspired by the algebraic parametric method, the modulating functions method can be extended to many applications. In [45], a fractional integration by parts formula has been obtained by working in the operational domain, then the modulating functions method has been generalized to fractional order systems identification problem. Inspired by [21, 22, 23], generalized modulating functions have been given [46], whose existence can be guarantied by the algebraic parametric method. Then, the modulating functions method has been generalized to design an integer order model-based differentiator [46], where the proposed differentiator does not contain any truncation error. Unlike the integer order model-based differentiators obtained with complex mathematical deduction in [22, 23], this differentiator is easy to obtain and to understand. Having these ideas in mind, the aim of this paper is to extend the modulating functions method to design a robust fractional order model-based differentiator without any truncation error. For this purpose, we will focus on a specific class of signals satisfying a linear differential equation with unknown parameters.

This paper is organized as follows. Section 2 begins with some basic definitions of fractional order derivatives. Then, a recent fractional integration by parts formula is recalled. In Section 3 , generalized modulating functions are first proposed. Then, the unknown parameters of the considered linear differential equation are estimated using a set of modulating functions. The generalized modulating functions and the fractional integration by parts formula are applied to obtain fractional order differentiators in continuous noise free case, which provide exact integral expressions for fractional order derivatives. Digital fractional order differentiators are deduced using the obtained integral expressions in discrete noisy case. Moreover, some error analysis results for noise error contribution are given. In Section 4, numerical results illustrate the accuracy and robustness of the proposed fractional order differentiators. Finally, some conclusions and perspectives are given in Section 5. Some classes of generalized modulating functions are presented in Appendix. 


\section{Preliminary}

\subsection{Riemann-Liouville and Caputo fractional derivatives}

Let $l \in \mathbb{N}, \alpha \in \mathbb{R} \backslash \mathbb{N}$ with $l-1<\alpha<l$, and $f \in \mathcal{C}^{l}(\mathbb{R})$ where $\mathcal{C}^{l}(\mathbb{R})$ refers to the set of functions being $l$-times continuously differentiable on $\mathbb{R}$. Then, we recall the following definitions.

Definition 1 ([3] p. 68) The left-sided Riemann-Liouville fractional derivative of $f$ is defined as follows: $\forall t \in[a,+\infty[$,

$$
{ }_{R} \mathrm{D}_{a, t}^{\alpha} f(t):=\frac{1}{\Gamma(l-\alpha)} \frac{d^{l}}{d t^{l}} \int_{a}^{t}(t-\tau)^{l-\alpha-1} f(\tau) d \tau,
$$

where $\Gamma(\cdot)$ is the Gamma function (see [47] p. 255).

Definition 2 ([3] p. 79) The left-sided Caputo fractional derivative of $f$ is defined as follows: $\forall t \in[a,+\infty[$,

$$
{ }_{C} \mathrm{D}_{a, t}^{\alpha} f(t):=\frac{1}{\Gamma(l-\alpha)} \int_{a}^{t}(t-\tau)^{l-\alpha-1} f^{(l)}(\tau) d \tau .
$$

Definition 3 ([4] p. 92) The right-sided Caputo fractional derivative of $f$ is defined as follows: $\forall t \in]-\infty, b]$,

$$
{ }_{C} \mathrm{D}_{t, b}^{\alpha} f(t):=\frac{(-1)^{l}}{\Gamma(l-\alpha)} \int_{t}^{b}(\tau-t)^{l-\alpha-1} f^{(l)}(\tau) d \tau .
$$

Consequently, if $-1<\alpha<l=0$, then we have ${ }_{R} \mathrm{D}_{a, t}^{\alpha} f(t) \equiv{ }_{C} \mathrm{D}_{a, t}^{\alpha} f(t)$. If $l \in \mathbb{N}^{*}\left(\alpha \in \mathbb{R}_{+} \backslash \mathbb{N}\right)$, by applying integration by parts to (1), we get (see [3] pp. 75-76): $\forall t \in[a,+\infty[$,

$$
{ }_{R} \mathrm{D}_{a, t}^{\alpha} f(t)=\sum_{i=0}^{l-1} \frac{(t-a)^{i-\alpha}}{\Gamma(i+1-\alpha)} f^{(i)}(a)+{ }_{C} \mathrm{D}_{a, t}^{\alpha} f(t) .
$$

In the following lemma, we give the right-sided Caputo fractional derivative of a polynomial.

Lemma 1 Let $f(\cdot)=(\cdot)^{n+l}$ with $l, n \in \mathbb{N}$, then the right-sided Caputo fractional derivative of $f$ is given by: $\forall t \in]-\infty, b]$,

$$
{ }_{C} \mathrm{D}_{t, b}^{\alpha} f(t)=\frac{(-1)^{l}}{\Gamma(l-\alpha)} \frac{(n+l) !}{n !} \sum_{i=0}^{n}\left(\begin{array}{c}
n \\
i
\end{array}\right) \frac{t^{n-i}(b-t)^{i+l-\alpha}}{i+l-\alpha},
$$

where $\alpha \in \mathbb{R} \backslash \mathbb{N}$ with $l-1<\alpha<l$.

Proof. By using (3), we get:

$$
{ }_{C} \mathrm{D}_{t, b}^{\alpha} f(t)=\frac{(-1)^{l}}{\Gamma(l-\alpha)} \frac{(n+l) !}{n !} \int_{t}^{b}(\tau-t)^{l-\alpha-1} \tau^{n} d \tau .
$$

Then, by taking the following change of variables $\tau \rightarrow \tau+t$, we obtain:

$$
{ }_{C} \mathrm{D}_{t, b}^{\alpha} f(t)=\frac{(-1)^{l}}{\Gamma(l-\alpha)} \frac{(n+l) !}{n !} \int_{0}^{b-t} \tau^{l-\alpha-1}(\tau+t)^{n} d \tau .
$$

Finally, this proof can be completed by applying the binomial theorem and calculating the integral in $(7)$. 


\subsection{Fractional integration by parts}

Integration by parts is a crucial tool for the use of modulating functions method which we are going to apply in this paper. For this reason, we recall the generalized integration by parts in the following lemma which can be obtained by recursively applying the classical integration by parts.

Lemma 2 Let $y, g \in \mathcal{C}^{l}(\mathbb{R})$ with $l \in \mathbb{N}^{*}$. Then, for any interval $[a, b] \subset \mathbb{R}$, we have:

$$
\int_{a}^{b} g(t) y^{(l)}(t) d t=\sum_{k=0}^{l-1}(-1)^{k}\left[g^{(k)}(t) y^{(l-1-k)}(t)\right]_{t=a}^{t=b}+(-1)^{l} \int_{a}^{b} g^{(l)}(t) y(t) d t .
$$

The previous formula has been extended to the fractional order case in [48]. Hence, fractional integration by parts formulae can be given in the following lemma.

Lemma 3 [48] Let $y, g \in \mathcal{C}^{l}(\mathbb{R})$ with $l \in \mathbb{N}$. Then, for any interval $[a, b] \subset \mathbb{R}$, we have:

$$
\int_{a}^{b} g(t)_{R} \mathrm{D}_{a, t}^{\alpha} y(t) d t=\int_{a}^{b}{ }_{C} \mathrm{D}_{t, b}^{\alpha} g(t) y(t) d t
$$

where $-1<\alpha<l=0$. Moreover, we have:

$$
\int_{a}^{b} g(t)_{R} \mathrm{D}_{a, t}^{\alpha} y(t) d t=\sum_{k=0}^{l-1}(-1)^{k}\left[g^{(k)}(t)_{R} \mathrm{D}_{a, t}^{\alpha-1-k} y(t)\right]_{t=a}^{t=b}+\int_{a}^{b} C_{C} \mathrm{D}_{t, b}^{\alpha} g(t) y(t) d t
$$

where $l \in \mathbb{N}^{*}$ and $l-1<\alpha<l$.

\section{Main result}

In this paper, we consider a class of signals satisfying the following differential equation:

$$
\forall t \in I, \sum_{i=0}^{N} a_{i} y^{(i)}(t)=u(t)
$$

where $y \in \mathcal{C}^{N}(I)$ with $N \in \mathbb{N}^{*}$ and $I=[a, b] \subset \mathbb{R}$. Moreover, $u$ is assumed to be known, and $a_{N} \in \mathbb{R}^{*}, a_{i} \in \mathbb{R}$, for $i=0, \ldots, N-1$, are unknown. In the case where $u \equiv 0$, we assume $a_{N}=1$. In the case where $u \neq 0$, we can consider $u$ as an input of a linear system defined by (11), and $y$ is the corresponding output.

In this section, we will propose fractional order differentiators for the left-sided RiemannLiouville fractional derivatives of $y$ both in continuous noise free case and in discrete noisy case. For this purpose, we begin with a generalization of classical modulating functions in the following subsection.

\subsection{Generalized modulating functions}

Definition 4 Let $[a, b] \subset \mathbb{R}, l, n \in \mathbb{N}$ with $n \leq l$, and $g$ be a function satisfying the following properties: 
$\left(P_{1}\right): g \in \mathcal{C}^{l+1}([a, b])$

$\left(P_{2}\right): g^{(i)}(a)=0$, for $i=0,1, \ldots, l$;

$\left(P_{3}\right): g^{(i)}(b)=0$, for $i=0,1, \ldots, n$, and $g^{(n+1)}(b) \neq 0$, if $n<l$.

Then, $g$ is called $(l, n)^{\text {th }}$ order modulating function on $[a, b]$ (see, [46]). If $g$ satisfies only the properties $\left(P_{1}\right)$ and $\left(P_{2}\right)$ for any $n \in \mathbb{N}$, i.e. $g(b) \neq 0$, then $g$ is called $(l,-1)^{\text {th }}$ order modulating function on $[a, b]$. If $l=n$, then $g$ is the classical $l^{\text {th }}$ order modulating function on $[a, b]$ (see, [41]). If $g$ satisfies the properties $\left(P_{1}\right),\left(P_{2}\right)$ and the following property:

$\left(P_{4}\right): g^{(i)}(b)=0$, for $i=0,1, \ldots, l$, except $i=n$.

Then, $g$ is called strict $(l, n-1)^{t h}$ order modulating function on $[a, b]$.

Consequently, if $g$ is a $(l-1)^{t h}$ order modulating function on $[a, b]$, then the boundary values in (8) and (10) can be canceled. If $g$ is a strict $(l-1, n-1)^{t h}$ order modulating function on $[a, b]$, then it remains only the boundary value $g^{(n)}(b) y^{(l-1-n)}(b)\left(\right.$ resp. $\left.g^{(n)}(b)_{R} \mathrm{D}_{a, b}^{\alpha-1-n} y(b)\right)$ in (8) (resp. (10)).

\subsection{Unknown parameter identification in continuous noise free case}

Before giving fractional order differentiators, we first calculate the unknown coefficients in (11). Modulating functions method is a classical identification method for continuous-time systems (see, e.g. $[40,41,42,43])$. The use of modulating functions allows to transform a differential equation of the analyzed signal into a sequence of algebraic integral equations. Then, unknown parameters can be estimated by solving a linear system. In the following proposition, we apply this method to give integral formulae for the unknown coefficients.

Proposition 1 [41] If $u \neq 0$ in (11), then let $g_{n}$, for $n=0, \ldots, L$, be a sequence of $(N-1)^{\text {th }}$ order modulating functions on $[t-T, t]$ with $N \leq L, a+T \leq t \leq b$ and $0<T \leq b-a$. The unknown coefficients can be given by solving the following linear system:

$$
Y_{t, T}\left(\begin{array}{c}
a_{0} \\
\vdots \\
a_{N}
\end{array}\right)=U_{t, T},
$$

where $Y_{t, T}(n+1, i+1)=(-1)^{i} \int_{t-T}^{t} g_{n}^{(i)}(\tau) y(\tau) d \tau$ and $U_{t, T}(n+1)=\int_{t-T}^{t} g_{n}(\tau) u(\tau) d \tau$, for $n=$ $0, \ldots, L$ and $i=0, \ldots, N$.

Proof. Step 1. Multiplication and integration: By multiplying both sides of (11) by $g_{n}$ and integrating from $t-T$ to $t$, we obtain: for $n=0, \ldots, L$,

$$
\sum_{i=0}^{N} a_{i} \int_{t-T}^{t} g_{n}(\tau) y^{(i)}(\tau) d \tau=\int_{t-T}^{t} g_{n}(\tau) u(\tau) d \tau
$$


Step 2. Integration by parts: By applying the generalized integration by parts given in Lemma 2 , we get: for $n=0, \ldots, L$,

$$
\sum_{i=0}^{N}(-1)^{i} a_{i} \int_{t-T}^{t} g_{n}^{(i)}(\tau) y(\tau) d \tau=\int_{t-T}^{t} g_{n}(\tau) u(\tau) d \tau
$$

where the boundary values are eliminated thanks to the properties of $g_{n}$. Consequently, this proof can be completed by solving the linear system given in (12).

We can see in Proposition 1 that by using the modulating functions method the unknown coefficients can be obtained using a sliding integration window of finite length. Moreover, we do not need to calculate the derivatives of $y$. Similarly, we can obtain the following corollary.

Corollary 1 If $u \equiv 0$ and $a_{N}=1$ in (11), then let $g_{n}$, for $n=0, \ldots, L-1$, be a sequence of $(N-1)^{t h}$ order modulating functions on $[t-T, t]$ with $N \leq L, a+T \leq t \leq b$ and $0<T \leq b-a$. The unknown coefficients can be given by solving the following linear system:

$$
\hat{Y}_{t, T}\left(\begin{array}{c}
a_{0} \\
\vdots \\
a_{N-1}
\end{array}\right)=\left(\begin{array}{c}
(-1)^{N+1} \int_{t-T}^{t} g_{0}^{(N)}(\tau) y(\tau) d \tau \\
\vdots \\
(-1)^{N+1} \int_{t-T}^{t} g_{L-1}^{(N)}(\tau) y(\tau) d \tau
\end{array}\right),
$$

where $\hat{Y}_{t, T}(n+1, i+1)=(-1)^{i} \int_{t-T}^{t} g_{n}^{(i)}(\tau) y(\tau) d \tau$, for $n=0, \ldots, L-1$ and $i=0, \ldots, N-1$.

Remark that if we apply generalized modulating functions in the proof of Proposition 1, then the boundary values at $\tau=t$ can be kept. Thus, the integer order derivatives of $y$ can be given. This is the idea of the generalized modulating functions for integer order numerical differentiation problem [46]. Bearing this idea in mind, we will give the fractional order derivatives of $y$ using generalized modulating functions in the next subsection.

\subsection{Exact fractional order differentiators in continuous noise free case}

In this subsection, we give fractional order differentiators in continuous noise free case. For this purpose, we first need to transfer the differential equation (11) into a fractional order differential equation.

Lemma 4 Let $y \in \mathcal{C}^{N}(I)$ with $N \in \mathbb{N}^{*}$ satisfy the differential equation given in (11), then we have:

$$
\forall t \in I, \sum_{i=0}^{N} a_{i} \mathrm{D}_{a, t}^{i-\beta} y(t)={ }_{R} \mathrm{D}_{a, t}^{-\beta} u(t)+\sum_{i=1}^{N} a_{i} \sum_{j=0}^{i-1} \frac{(t-a)^{j-i+\beta}}{\Gamma(j+1-i+\beta)} y^{(j)}(a),
$$

where $0<\beta<1$.

Proof. By using (1) with $l=0$ and $\beta=-\alpha$, we can take the $-\beta^{\text {th }}$ order left-sided RiemannLiouville fractional derivative to (11). Thus, we obtain:

$$
\forall t \in I, \sum_{i=0}^{N} a_{i} \frac{1}{\Gamma(\beta)} \int_{a}^{t}(t-\tau)^{\beta-1} y^{(i)}(\tau) d \tau=\frac{1}{\Gamma(\beta)} \int_{a}^{t}(t-\tau)^{\beta-1} u(t) d \tau .
$$


Then, according to (2), (17) becomes:

$$
\forall t \in I, \sum_{i=0}^{N} a_{i} \mathrm{D}_{a, t}^{i-\beta} y(t)={ }_{R} \mathrm{D}_{a, t}^{-\beta} u(t) .
$$

Finally, this proof can be completed using (4).

Now, by using generalized modulating functions and the fractional integration by parts, we can give the following theorem.

Theorem 1 Let $h_{n}$, for $n=0, \ldots, N-1$, be a sequence of strict $(N-1, n-1)^{\text {th }}$ order modulating functions on $[a, t]$ with $t \in I$. If $h_{n}$ also satisfies the following property:

$\left(P_{5}\right): \int_{a}^{t}(\tau-a)^{\beta-i} h_{n}(\tau) d \tau=0$, for $i=1,2, \ldots, N$, with $0<\beta<1$.

Then, the left-sided Riemann-Liouville fractional derivatives of $y$ is given by: $\forall t \in I$,

$$
{ }_{R} \mathrm{D}_{a, t}^{-\beta} y(t)=\frac{(-1)^{N-1}}{h_{N-1}^{(N-1)}(t) a_{N}}\left(\int_{a}^{t}{ }_{C} \mathrm{D}_{\tau, t}^{-\beta} h_{N-1}(\tau) u(\tau) d \tau-\sum_{i=0}^{N} a_{i} \int_{a}^{t}{ }_{C} \mathrm{D}_{\tau, t}^{i-\beta} h_{N-1}(\tau) y(\tau) d \tau\right),
$$

and for $n=N-2, N-3, \ldots, 0$,

$$
\begin{gathered}
{ }_{R} \mathrm{D}_{a, t}^{N-n-1-\beta} y(t)=\frac{(-1)^{n}}{h_{n}^{(n)}(t) a_{N}} \int_{a}^{t}{ }_{C} \mathrm{D}_{\tau, t}^{-\beta} h_{n}(\tau) u(\tau) d \tau-\sum_{i=n+1}^{N-1} \frac{a_{i}}{a_{N}}{ }_{R} \mathrm{D}_{a, t}^{i-n-1-\beta} y(t) \\
+\frac{1}{h_{n}^{(n)}(t) a_{N}} \sum_{i=0}^{N}(-1)^{n+1} a_{i} \int_{a}^{t}{ }_{C} \mathrm{D}_{\tau, t}^{i-\beta} h_{n}(\tau) y(\tau) d \tau,
\end{gathered}
$$

where $a_{i}$, for $i=0, \ldots, N$, are given by Proposition 1 or Corollary 1.

Remark 1 If the order $\alpha$ of the derivative which we want to estimate is larger than $N$, then in order to apply Theorem 1 we need to differentiate Equation (11) $(\lceil\alpha\rceil-N)$ times, where $\lceil\alpha\rceil$ denotes the smallest integer greater than $\alpha$.

Proof of Theorem 1. Step 1. Multiplication and integration: By multiplying both sides of (16) by $h_{n}$ and integrating from $a$ to $t$, we obtain: for $n=0, \ldots, N-1, \forall t \in I$,

$$
\sum_{i=0}^{N} a_{i} \int_{a}^{t} h_{n}(\tau)_{R} \mathrm{D}_{a, \tau}^{i-\beta} y(\tau) d \tau=\int_{a}^{t} h_{n}(\tau)_{R} \mathrm{D}_{a, \tau}^{-\beta} u(\tau) d \tau+\sum_{i=1}^{N} a_{i} \sum_{j=0}^{i-1} y^{(j)}(a) \int_{a}^{t} \frac{h_{n}(\tau)(\tau-a)^{j-i+\beta}}{\Gamma(j+1-i+\beta)} d \tau .
$$

Step 2. Elimination of all the initial conditions: Since $h_{n}$ satisfies the property $\left(P_{5}\right)$, all the unknown initial conditions $y^{(j)}(a)$ can be eliminated in (21). Thus, we get: $\forall t \in I$,

$$
\sum_{i=0}^{N} a_{i} \int_{a}^{t} h_{n}(\tau)_{R} \mathrm{D}_{a, \tau}^{i-\beta} y(\tau) d \tau=\int_{a}^{t} h_{n}(\tau)_{R} \mathrm{D}_{a, \tau}^{-\beta} u(\tau) d \tau .
$$


Step 3. Fractional integration by parts: By applying the fractional integration by parts given in Lemma 3, we get: $\forall t \in I$,

$$
\begin{aligned}
\int_{a}^{t}{ }_{C} \mathrm{D}_{\tau, t}^{-\beta} h_{n}(\tau) u(\tau) d \tau= & \sum_{i=0}^{N} a_{i} \int_{a}^{t} C_{C, t} \mathrm{D}_{\tau, t}^{i-\beta} h_{n}(\tau) y(\tau) d \tau \\
& +\sum_{i=1}^{N} a_{i} \sum_{k=0}^{i-1}(-1)^{k}\left[h_{n}^{(k)}(\tau)_{R} \mathrm{D}_{a, \tau}^{i-\beta-1-k} y(\tau)\right]_{\tau=a}^{\tau=t}
\end{aligned}
$$

Step 4. Elimination of all the boundary values at $\tau=a$ : Since $h_{n}$ satisfies the property $\left(P_{2}\right)$ with $l=N-1$, we have $h_{n}^{(i)}(a)=0$, for $i=0, \ldots, N-1$. Hence, $(23)$ becomes: $\forall t \in I$,

$$
\begin{aligned}
\int_{a}^{t}{ }_{C} \mathrm{D}_{\tau, t}^{-\beta} h_{n}(\tau) u(\tau) d \tau=\sum_{i=0}^{N} & a_{i} \int_{a}^{t}{ }_{C} \mathrm{D}_{\tau, t}^{i-\beta} h_{n}(\tau) y(\tau) d \tau \\
& +\sum_{i=1}^{N} a_{i} \sum_{k=0}^{i-1}(-1)^{k} h_{n}^{(k)}(t)_{R} \mathrm{D}_{a, t}^{i-\beta-1-k} y(t) .
\end{aligned}
$$

Then, all the boundary values at $\tau=t$ in (24) can be given in the following matrix:

$$
\left(\begin{array}{cccc}
a_{1} h_{n}^{(0)}(t)_{R} \mathrm{D}_{a, t}^{-\beta} y(t) & a_{2} h_{n}^{(0)}(t)_{R} \mathrm{D}_{a, t}^{1-\beta} y(t) & \cdots & a_{N} h_{n}^{(0)}(t)_{R} \mathrm{D}_{a, t}^{N-1-\beta} y(t) \\
0 & -a_{2} h_{n}^{(1)}(t)_{R} \mathrm{D}_{a, t}^{-\beta} y(t) & \cdots & -a_{N} h_{n}^{(1)}(t)_{R} \mathrm{D}_{a, t}^{N-2-\beta} y(t) \\
0 & 0 & \cdots & (-1)^{2} a_{N} h_{n}^{(2)}(t)_{R} \mathrm{D}_{a, t}^{N-3-\beta} y(t) \\
\vdots & \vdots & \ddots & \vdots \\
0 & 0 & \cdots & (-1)^{N-1} a_{N} h_{n}^{(N-1)}(t)_{R} \mathrm{D}_{a, t}^{-\beta} y(t)
\end{array}\right) .
$$

Step 5. Calculation of the boundary values at $\tau=t$ : Since $h_{n}$ satisfies the property $\left(P_{4}\right)$ with $l=$ $N-1$, we have $h_{n}^{(i)}(t)=0$, for $i=0, \ldots, N-1$, except $i=n$. Hence, all the rows in (25) are equal to 0 , except the $(n+1)^{t h}$ row. Thus, $(24)$ becomes: for $n=0, \ldots, N-1, \forall t \in I$,

$$
\int_{a}^{t}{ }_{C} \mathrm{D}_{\tau, t}^{-\beta} h_{n}(\tau) u(\tau) d \tau=\sum_{i=0}^{N} a_{i} \int_{a}^{t} C \mathrm{D}_{\tau, t}^{i-\beta} h_{n}(\tau) y(\tau) d \tau+(-1)^{n} h_{n}^{(n)}(t) \sum_{i=n+1}^{N} a_{i R} \mathrm{D}_{a, t}^{i-n-1-\beta} y(t) .
$$

Consequently, this proof can be completed using (26).

We can see in Theorem 1 that the fractional order derivatives of $y$ are simultaneously given in a recursive way. Inspired by Theorem 1, we can obtain the following corollary, where the fractional order derivatives of $y$ are independently given.

Corollary 2 Let $f_{n}$, for $n=0, \ldots, N-1$, be a sequence of functions satisfying the properties $\left(P_{1}\right),\left(P_{2}\right)$ and $\left(P_{5}\right)$ on $[a, t]$ with $l=N-1$ and $t \in I$. If $f_{n}$ also satisfies the following property:

$\left(P_{6}\right): F_{i}(t):=\sum_{j=0}^{N-1-i}(-1)^{j} a_{i+1+j} f_{i}^{(j)}(t)=0$, for $i=0,1, \ldots, N-1$, except $i=n$.

Then, the left-sided Riemann-Liouville fractional derivatives of $y$ can be given by: for $n=0,1, \ldots, N-$ $1, \forall t \in I$,

$$
{ }_{R} \mathrm{D}_{a, t}^{n-\beta} y(t)=\frac{1}{F_{n}(t)}\left(\int_{a}^{t}{ }_{C} \mathrm{D}_{\tau, t}^{-\beta} f_{n}(\tau) u(\tau) d \tau-\sum_{i=0}^{N} a_{i} \int_{a}^{t}{ }_{C} \mathrm{D}_{\tau, t}^{i-\beta} f_{n}(\tau) y(\tau) d \tau\right)
$$


where $0<\beta<1, a_{i}$, for $i=0, \ldots, N$, are given by Proposition 1 or Corollary 1.

The proof of Corollary 2 is similar to the one of Theorem 1, except Step 5 . The property $\left(P_{6}\right)$ allows us to eliminate all the undesired boundary values at $\tau=t$ in the diagonals of (25). In Appendix, we will give two classes of generalized modulating functions satisfying respectively the properties required in Theorem 1 and Corollary 2.

According to Theorem 1 and Corollary 2, the left-sided Riemann-Liouville fractional derivatives of $y$ are exactly given by integral formulae. Unlike the improper integral in the definition of the left-sided Riemann-Liouville fractional given in (1), the proposed integral formulae can be proper by choosing appropriate modulating functions. Consequently, the proposed fractional order differentiators do not contain any source of errors in continuous noise free case. Moreover, they can be used for on-line applications.

Similarly, if we apply $f_{n}(a+t-\cdot)$, for $n=0, \ldots, N-1$, as generalized modulating functions on $[a, t]$ in the proof of Theorem 1 , where $f_{n}(\cdot)$ is given by Corollary 2, then the initial conditions at $\tau=a$ can be given by integral formulae. Then, by using Corollary 2 and Equation (4), the left-sided Caputo fractional derivatives of $y$ can also be given. This is out of the scope of this paper.

\subsection{Digital fractional order differentiators in noisy case}

From now on, let $y^{\varpi}$ be a discrete noisy observation of $y$ on $I=[a, b]$ :

$$
y^{\varpi}\left(t_{i}\right)=y\left(t_{i}\right)+\varpi\left(t_{i}\right),
$$

where $t_{i}=a+i T_{s}$, for $i=0,1, \ldots, M$, with an equidistant sampling period $T_{s}=\frac{b-a}{M}$. Moreover, the noise $\{\varpi(t), t \in I\}$ is assumed to be a continuous stochastic process satisfying the following conditions:

$\left(C_{1}\right)$ : for any $s, t \in I, s \neq t, \varpi(s)$ and $\varpi(t)$ are independent;

$\left(C_{2}\right)$ : the mean value function of $\{\varpi(t), t \in I\}$ denoted by $\mathbb{E}[\cdot]$ is equal to zero;

$\left(C_{3}\right)$ : the variance function of $\{\varpi(t), t \in I\}$ denoted by $\operatorname{Var}[\cdot]$ is bounded on $I$, i.e. $\exists \delta \in \mathbb{R}_{+}, \forall t \in$ $I, \operatorname{Var}[\varpi(t)] \leq \delta$.

Note that a zero-mean white Gaussian noise satisfies these conditions.

In order to estimate the left-sided Riemann-Liouville fractional derivatives of $y$ using its discrete noisy observation $y^{\varpi}$, we apply the integral formulae given in Theorem 1 and Corollary 2 . Since $y^{\varpi}$ is a discrete measurement and the integrals in the formulae can be proper, we apply a numerical integration method to approximate the integrals so as to obtain digital fractional order differentiators. Let us denote the integrals involving $y$ in Equations (19), (20) and (27) as follows:

$$
I_{q}^{y}(t):=\int_{a}^{t} q(\tau) y(\tau) d \tau
$$


where $q$ denotes the right-sided Caputo fractional derivatives of the used modulating functions. Hence, $I_{q}^{y}$ can be approximated in the discrete case by:

$$
I_{q}^{y, i}:=T_{s} \sum_{j=0}^{i} w_{j} q\left(t_{j}\right) y\left(t_{j}\right),
$$

where $w_{i} \in \mathbb{R}_{+}$are the weights for a given numerical integration method. By using the discrete noisy observation $y^{\varpi}$, we get:

$$
I_{q}^{y^{\varpi}, i}=I_{q}^{y, i}+e_{q}^{\varpi, i}
$$

where $e_{q}^{\varpi, i}:=T_{s} \sum_{j=0}^{i} w_{j} q\left(t_{j}\right) \varpi\left(t_{j}\right)$. Thus, the integral $I_{q}^{y}(t)$ is subject to two sources of errors:

- the numerical error due to the numerical integration method;

- the noise error contribution $e_{q}^{\varpi, i}$.

Similarly, we can deduce that the integral formulae given in Proposition 1 and Corollary 1 for the unknown coefficients also contain these two sources of errors. Consequently, the proposed digital fractional order differentiators contain numerical errors and noise error contributions in the discrete noisy case. It is well known that the numerical error converges to zero when $T_{s} \rightarrow 0$. In the following proposition, we show the convergence in mean square of the noise error contribution when $T_{s} \rightarrow 0$.

Proposition 2 Let $\left\{\varpi\left(t_{i}\right)\right\}$ be a sequence of $\{\varpi(t), t \in I\}$ with an equidistant sampling period $T_{s}$, where $\{\varpi(t), t \in I\}$ is a continuous stochastic process satisfying conditions $\left(C_{1}\right)-\left(C_{3}\right)$. Assume that $q \in \mathcal{L}^{2}([a, t])$, then we have:

$$
\mathbb{E}\left[\left(e_{q}^{\varpi, i}\right)^{2}\right] \underset{T_{s} \rightarrow 0}{\longrightarrow} 0
$$

where $e_{q}^{\varpi, i}$ is given by (31).

The proof of the previous proposition can be obtained using a similar way to Theorem 1 in [36]. In fact, using $\left(C_{1}\right),\left(C_{2}\right)$ and $\left(C_{3}\right)$ we obtain: $\mathbb{E}\left[e_{q}^{\varpi, i}\right]=0$ and

$$
\mathbb{E}\left[\left(e_{q}^{\varpi, i}\right)^{2}\right]=\operatorname{Var}\left[e_{q}^{\varpi, i}\right] \leq T_{s} \delta\left(T_{s} \sum_{j=0}^{i} w_{j}^{2} q^{2}\left(t_{j}\right)\right) .
$$

Hence, if $q \in \mathcal{L}^{2}([a, t])$ then the convergence in Equation (32) can be obtained. A similar result has been shown using non-standard analysis in [24, 25]. Consequently, we can reduce both numerical errors and noise error contributions in the proposed digital fractional order differentiators by decreasing the sampling period $T_{s}$.

When the sampling period is set, on the one hand the numerical error can be bounded by the well-known error bounds for numerical integration methods [49], on the other hand the noise error 
contribution $e_{q}^{\varpi, i}$ satisfying $\mathbb{E}\left[e_{q}^{\varpi, i}\right]=0$ can be bounded using the Bienaymé-Chebyshev inequality:

$$
\forall \gamma \in \mathbb{R}_{+}^{*}, \operatorname{Pr}\left(\left|e_{q}^{\varpi, i}\right|<\gamma\left(\operatorname{Var}\left[e_{q}^{\varpi, i}\right]\right)^{\frac{1}{2}}\right)>1-\frac{1}{\gamma^{2}},
$$

i.e. the probability for $\left|e_{q}^{\varpi, i}\right|$ to be smaller than $\gamma\left(\operatorname{Var}\left[e_{q}^{\varpi, i}\right]\right)^{\frac{1}{2}}$ is larger than $1-\frac{1}{\gamma^{2}}$. Thus, we deduce from (33) and (34) the following error bound:

$$
\left|e_{q}^{\varpi, i}\right| \stackrel{p_{\gamma}}{<} \gamma T_{s}\left(\delta \sum_{j=0}^{i} w_{j}^{2} q^{2}\left(t_{j}\right)\right)^{\frac{1}{2}},
$$

where $a \stackrel{p_{\gamma}}{<} b$ means that the probability for a real number $b$ to be larger than an other real number $a$ is equal to $p_{\gamma}$ with $p_{\gamma}>1-\frac{1}{\gamma^{2}}$. Remark that the value of $p_{\gamma}$ can be given by the probability density function of the considered noise $\varpi$. In particular, if $\varpi$ is a white Gaussian noise, then according to the three-sigma rule, we have:

$$
\left|e_{q}^{\varpi, i}\right| \stackrel{p_{\gamma}}{\leq} \gamma T_{s}\left(\delta \sum_{j=0}^{i} w_{j}^{2} q^{2}\left(t_{j}\right)\right)^{\frac{1}{2}},
$$

where $p_{1}=68.26 \%, p_{2}=95.44 \%$ and $p_{3}=99.73 \%$, for $\gamma=1,2,3$, respectively.

\section{Simulation results}

In this section, we give some numerical results to show the accuracy and robustness of the proposed digital fractional order differentiators. We assume that $y^{\varpi}\left(t_{i}\right)=y\left(t_{i}\right)+\varpi\left(t_{i}\right)$ is a discrete noisy observation defined in (28) with $I=[0,10]$ and $T_{s}=10^{-3}$, where the noise $\left\{\varpi\left(t_{i}\right)\right\}$ is simulated from a zero-mean white Gaussian iid sequence, and the variance is adjusted such that the signal-to-noise ratio $\mathrm{SNR}=10 \log _{10}\left(\frac{\sum\left|y^{\varpi}\left(t_{i}\right)\right|^{2}}{\sum\left|\varpi\left(t_{i}\right)\right|_{2}^{2}}\right)$ is equal to $\mathrm{SNR}=\rho \mathrm{dB}$ with $\rho \in \mathbb{R}_{+}^{*}$. In the following subsections, we assume $y$ is a polynomial signal and a sinusoidal signal respectively.

\subsection{Polynomial signal}

In this subsection, we consider a second degree polynomial given by:

$$
\forall t \in I, y(t)=p_{2} t^{2}+p_{1} t+p_{0}
$$

where $p_{2}, p_{1}, p_{0} \in \mathbb{R}_{+}^{*}$. Hence, $y$ satisfies the following third order differential equation:

$$
\forall t \in I, y^{(3)}(t)=0
$$

Moreover, the left-side Riemann-Liouville fractional derivative of $y$ is given using the following formula ([3], p. 72 ): for $\alpha<n$ with $\alpha \in \mathbb{R}_{+} \backslash \mathbb{N}$,

$$
\forall t \in I,{ }_{R} \mathrm{D}_{0, t}^{\alpha} t^{n}=\frac{\Gamma(n+1)}{\Gamma(n+1-\alpha)} t^{n-\alpha} .
$$


Since there is no unknown parameter in (38), we can directly apply Theorem 1 . Then, we obtain: $\forall t \in I$,

$$
\begin{aligned}
& { }_{R} \mathrm{D}_{0, t}^{1-\beta} y(t)=\frac{1}{h_{1}^{(1)}(t)} \int_{0}^{t}{ }_{C} \mathrm{D}_{\tau, t}^{3-\beta} h_{1}(\tau) y(\tau) d \tau, \\
& { }_{R} \mathrm{D}_{0, t}^{2-\beta} y(t)=-\frac{1}{h_{0}(t)} \int_{0}^{t}{ }_{C} \mathrm{D}_{\tau, t}^{3-\beta} h_{0}(\tau) y(\tau) d \tau,
\end{aligned}
$$

where $0<\beta<1, h_{0}$ and $h_{1}$ are given by Proposition 3 in Appendix with $N=3$. Consequently, we can estimate ${ }_{R} \mathrm{D}_{0, t}^{\alpha} y(t)$ for any $\left.\alpha \in\right] 0,1[\cup] 1,2[$.

Example 1. In this example, we take $p_{2}=p_{0}=1, p_{1}=-10$ and $\rho=30(\mathrm{SNR}=30 \mathrm{~dB})$. The corresponding $y$ and $y^{\varpi}$ are shown in Fig. 1 .

We are going to estimate ${ }_{R} \mathrm{D}_{0, t_{i}}^{0.5} y\left(t_{i}\right)$ and ${ }_{R} \mathrm{D}_{0, t_{i}}^{1.5} y\left(t_{i}\right)$ for $t_{i} \in[0.5,10]$. For this purpose, we apply the trapezoidal numerical integration method to approximate the integrals in (40) and (41) by considering the noisy observation $y^{\varpi}$. The obtained estimations and the corresponding absolute estimation errors are given in Fig. 2 and Fig. 3 respectively. We give in Fig. 4 the absolute estimation errors obtained in noise free case, which are only due to the numerical errors. Consequently, we can see that the noise error contributions are relatively large in the beginning. When $t$ is larger than 4 , we have good results both in the noisy case and in noise free case. Moreover, we can observe that the numerical errors are much smaller than the noise error contributions.

\subsection{Sinusoidal signal}

In this subsection, we consider a sinusoidal signal in the following form:

$$
\forall t \in I, y(t)=A \sin (\omega t+\phi),
$$

where the parameters $A \in \mathbb{R}_{+}^{*}, \omega \in \mathbb{R}_{+}^{*}$ and $-\frac{1}{2} \pi<\phi<\frac{1}{2} \pi$ are unknown. Hence, $y$ satisfies the following harmonic oscillator equation:

$$
\forall t \in I, \ddot{y}(t)+\omega^{2} y(t)=0 .
$$

Moreover, the left-side Riemann-Liouville fractional derivative of $y$ can be given using the expansion of $y$ and the linearity of the Riemann-Liouville fractional derivative ([3], p. 91):

$$
\forall t \in I,{ }_{R} \mathrm{D}_{0, t}^{\alpha} y(t)=A \cos \phi_{R} \mathrm{D}_{0, t}^{\alpha} \sin (\omega t)+A \sin \phi_{R} \mathrm{D}_{0, t}^{\alpha} \cos (\omega t)
$$

with

$$
\begin{aligned}
& { }_{R} \mathrm{D}_{0, t}^{\alpha} \sin (\omega t)=\frac{\omega t^{1-\alpha}}{\Gamma(2-\alpha)}{ }_{1} \mathrm{~F}_{2}\left(1 ; \frac{1}{2}(2-\alpha), \frac{1}{2}(3-\alpha) ;-\frac{1}{4} \omega^{2} t^{2}\right), \\
& { }_{R} \mathrm{D}_{0, t}^{\alpha} \cos (\omega t)=\frac{t^{-\alpha}}{\Gamma(1-\alpha)}{ }_{1} \mathrm{~F}_{2}\left(1 ; \frac{1}{2}(1-\alpha), \frac{1}{2}(2-\alpha) ;-\frac{1}{4} \omega^{2} t^{2}\right),
\end{aligned}
$$


where ${ }_{1} \mathrm{~F}_{2}$ is the generalized hypergeometric function (see [50] p. 83, p. 303 and pp. 317-318).

Step 1. Estimation of the unknown frequency: According to Corollary 1, the unknown frequency $\omega^{2}$ can be given using a sliding integration window as follows:

$$
\forall t \in[T, b], \omega^{2}=-\frac{\int_{t-T}^{t} \ddot{g}_{t, T}(\tau) y(\tau) d \tau}{\int_{t-T}^{t} g_{t, T}(\tau) y(\tau) d \tau},
$$

where $g_{t, T}$ is a first order modulating function on $[t-T, t]$ with $0<T \leq b$. Then, it is estimated in the discrete noisy case by: for $i=m, \ldots, M$,

$$
\tilde{\omega}^{2}\left(t_{i}\right):=-\frac{I_{\ddot{g}_{t_{i}, T}}^{y^{\varpi}}}{I_{g_{t_{i}, T}}^{y^{\varpi}}},
$$

where $T=m T_{s}$ with $1 \leq m \leq M, I_{\ddot{g}_{t_{i}, T}}^{y^{\varpi}}$ and $I_{g_{t_{i}, T}}^{y^{\varpi}}$ are respectively the approximations of the integrals obtained in (47) by applying the trapezoidal numerical integration method with the noisy observation $y^{\varpi}$. Similar estimators have also been obtained using the algebraic parametric method in $[32,37]$.

Remark that if $I_{g_{t}, T}^{y^{\varpi}}=0$, then there is a singular value in (48). In order to solve this problem, we use the following weighted least square criterion as done in [44]: for $i=m, \ldots, M$,

$$
\min _{\theta_{i} \in \mathbb{R}} J\left(\theta_{i}\right):=\frac{1}{2} \sum_{j=m}^{i} \nu^{i+1-j}\left(I_{\ddot{g}_{t_{j}, T}}^{y^{\varpi}}+I_{g_{t_{j}, T}}^{y^{\varpi}} \theta_{i}\right)^{2},
$$

where $\theta_{i}=\tilde{\omega}^{2}\left(t_{i}\right)$, and $\left.\left.\nu \in\right] 0,1\right]$ represents a forgetting factor to exponentially discard the "old" data in the recursive schema. By solving $\frac{d J\left(\theta_{i}\right)}{d \theta_{i}}=0$, we can find the value of $\theta_{i}$ which minimizes the criterion (49): for $i=m, \ldots, M$,

$$
\theta_{i}=-\frac{\sum_{j=m}^{i} \nu^{i+1-j} I_{\ddot{g}_{t_{j}, T}}^{y^{\varpi}} I_{g_{t_{j}, T}}^{y^{\varpi}}}{\sum_{j=m}^{i} \nu^{i+1-j}\left(I_{g_{t_{j}, T}}^{y^{\varpi}}\right)^{2}} .
$$

Moreover, by using a similar way as done in [44], we obtain the following recursive algorithm:

$$
\theta_{i+1}=\frac{\nu}{\mu_{i+1}}\left(\mu_{i} \theta_{i}-I_{\ddot{g}_{t_{i+1}, T}}^{y^{\varpi}} I_{g_{t_{i+1}, T}}^{y^{\varpi}}\right), i=m, \ldots, M,
$$

where $\mu_{i}=\sum_{j=m}^{i} \nu^{i+1-j}\left(I_{g_{t_{j}, T}}^{y^{\varpi}}\right)^{2}$ can be recursively calculated by $\mu_{i+1}=\nu\left(\mu_{i}+\left(I_{g_{t_{i+1}, T}}^{y^{\varpi}}\right)^{2}\right)$.

Step 2. Estimation of the fractional order derivatives: We want to estimate ${ }_{R} \mathrm{D}_{0, t}^{\alpha} y(t)$ for any $\alpha \in$ ]0,1[U]1,2[. According to Remark 1, we need to take once differentiation to (43). Hence, we get:

$$
\forall t \in I, y^{(3)}(t)+\omega^{2} y^{(1)}(t)=0 .
$$


Then, by applying Corollary 2, we obtain:

$$
\begin{aligned}
& { }_{R} \mathrm{D}_{0, t}^{1-\beta} y(t)=\frac{1}{f_{1}^{(1)}(t)}\left(\int_{0}^{t}{ }_{C} \mathrm{D}_{\tau, t}^{3-\beta} f_{1}(\tau) y(\tau) d \tau+\omega^{2} \int_{0}^{t}{ }_{C} \mathrm{D}_{\tau, t}^{1-\beta} f_{1}(\tau) y(\tau) d \tau\right) \\
& { }_{R} \mathrm{D}_{0, t}^{2-\beta} y(t)=-\frac{1}{f_{2}(t)}\left(\int_{0}^{t}{ }_{C} \mathrm{D}_{\tau, t}^{3-\beta} f_{2}(\tau) y(\tau) d \tau+\omega^{2} \int_{0}^{t}{ }_{C} \mathrm{D}_{\tau, t}^{1-\beta} f_{2}(\tau) y(\tau) d \tau\right)
\end{aligned}
$$

where $0<\beta<1, f_{1}$ and $f_{2}$ are given by Corollary 3 in Appendix with $N=3, a_{3}=1, a_{1}=\omega^{2}$ and $a_{2}=a_{0}=0$. Consequently, we can estimate ${ }_{R} \mathrm{D}_{0, t}^{\alpha} y(t)$ for any $\left.\alpha \in\right] 0,1[\cup] 1,2[$.

Example 2. In this example, we take $A=3, \omega=5, \phi=0.5$ and $\rho=15(\mathrm{SNR}=15 \mathrm{~dB})$. The corresponding $y$ and $y^{\varpi}$ are shown in Fig. 5(a). The trapezoidal numerical integration method is also applied to approximate the integrals with the noisy observation $y^{\varpi}$. We first estimate the unknown frequency using (51) with $T=0.5$ and $\nu=1$. The obtained relative error is given in Fig. $5(\mathrm{~b})$. Then, we estimate ${ }_{R} \mathrm{D}_{0, t_{i}}^{0.5} y\left(t_{i}\right)$ and ${ }_{R} \mathrm{D}_{0, t_{i}}^{1.5} y\left(t_{i}\right)$ for $t_{i} \in[0.5,10]$. The obtained estimations and the corresponding absolute estimation errors are given in Fig. 6 and Fig. 7 respectively. We give in Fig. 8 the absolute estimation errors obtained in the noise free case, which are due to the numerical errors. Consequently, we can see that the noise error contributions are relatively large in the beginning. When $t$ becomes larger, we have good results both in the noisy case and in noise free case. Moreover, as shown in the previous example, the numerical errors are much smaller than the noise error contributions. However, since the used modulating functions are polynomials, the noise error contributions can increase with respect to $t$. In order to solve this problem, the Short-Memory Principe can be applied (see [3], p. 203). Otherwise, we can consider other types of modulating functions. This is out the scope of this paper.

\section{Conclusions}

In this paper, robust fractional order differentiators have been proposed in the time domain for a class of signals satisfying a linear differential equation with unknown parameters. We have first generalized the classical modulating functions method which has been used to estimate the unknown parameters. Then, using two different classes of generalized modulating functions and the fractional integration by parts formula, integral formulae have been derived for the estimation of left-sided Riemann-Liouville fractional derivative of the considered signal in two different ways. These formulae have been considered as proposed fractional order differentiators which do not contain any sources of errors in continuous noise free case. Digital fractional order differentiators have been deduced using a numerical integration method in discrete noisy case. They can be used for on-line applications to estimate the left-sided Riemann-Liouville fractional derivative with an arbitrary order. There are two sources of errors for these digital fractional order differentiators: numerical errors and noise error contributions. On the one hand, since the integrals in the proposed 
fractional order differentiators can be proper by choosing appropriate modulating functions, the numerical errors can be much smaller than the noise error contributions. On the other hand, since the integrals can be considered as a low-pass filter, it has been shown that the noise error contributions due to a class of stochastic processes can be reduced when the sampling period decreases. Moreover, some error bounds have been given for the noise error contributions. Finally, two numerical examples have been given to show the accuracy and robustness of the proposed fractional order differentiators. In these examples, we have only considered polynomials as generalized modulating functions which have been constructed in Appendix. The other types of generalized modulating functions and their influences to the noise error contributions will be studied in a future work.

\section{Appendix}

There exists several kinds of modulating functions in the literatures (see, e.g. [41]). Unlike classical integer order derivatives, the fractional order derivatives of the existing modulating functions usually cannot be formally calculated. For this reason, we consider polynomials whose fractional order derivatives are easy to calculate. Moreover, inspired by the algebraic parametric method (see, e.g. $[22,23]$ ), it is easy to find polynomials satisfying the properties of generalized modulating functions.

Proposition 3 Let $[a, t] \subset \mathbb{R}, n \in \mathbb{N}, N \in \mathbb{N}^{*}$ with $n \leq N-1$, and $h_{n}$ be a polynomial with the following form:

$$
h_{n}(\tau)=\sum_{i=0}^{2 N-1} c_{i} t^{2 N-1-i}(\tau-a)^{N+i},
$$

where the coefficient vector $\left(c_{0}, \ldots, c_{2 N-1}\right)^{T}$ is the solution of the following system:

$$
A_{n}\left(\begin{array}{c}
c_{0} \\
\vdots \\
c_{2 N-1}
\end{array}\right)=I_{n}
$$

where for $j=0, \ldots, 2 N-1, I_{n}(n)=1, I_{n}(j)=0$ with $j \neq n$, and for $i=0, \ldots, 2 N-1$,

$$
A_{n}(j, i)= \begin{cases}\frac{(N+i) !}{(N+i-j) !} t^{2 N-1-i}(t-a)^{N+i-j}, & \text { for } j=0, \ldots, N-1, \\ \frac{1}{2 N+i+\beta-j} t^{2 N-1-i}(t-a)^{2 N+i+\beta-j}, & \text { for } j=N, \ldots, 2 N-1 .\end{cases}
$$

Then, $h_{n}$ is a strict $(N-1, n-1)^{\text {th }}$ order modulating function satisfying the property $\left(P_{5}\right)$ on $[a, t]$.

Proof. By calculating the $j^{\text {th }}$ order derivative of $h_{n}$, for $j=0, \ldots, N-1$, we obtain:

$$
h_{n}^{(j)}(\tau)=\sum_{i=0}^{2 N-1} c_{i} \frac{(N+i) !}{(N+i-j) !} t^{2 N-1-i}(\tau-a)^{N+i-j}
$$


Hence, $h_{n}$ satisfies the properties $\left(P_{1}\right)$ and $\left(P_{2}\right)$ on $[a, t]$ with $l=N-1$. Then, we calculate the following integral: for $j=1, \ldots, N$,

$$
\int_{a}^{t}(\tau-a)^{\beta-j} h_{n}(\tau) d \tau=\sum_{i=0}^{2 N-1} c_{i} \frac{t^{2 N-1-i}(t-a)^{N+i+\beta-j+1}}{N+i+\beta-j+1} .
$$

Consequently, if $\left(c_{0}, \ldots, c_{2 N-1}\right)^{T}$ is the solution of the system (56), then $h_{n}$ satisfies also the properties $\left(P_{4}\right)$ and $\left(P_{5}\right)$ on $[a, t]$.

Let us remark that if $a=0$ in Proposition 3, then the power of $t$ in each row of $A_{n}$ is equal. Hence, $A_{n}$ can be simplified by the following form:

$$
\hat{A}_{n}(j, i)= \begin{cases}\frac{(N+i) !}{(N+i-j) !}, & \text { for } j=0, \ldots, N-1, \\ \frac{1}{2 N+i+\beta-j}, & \text { for } j=N, \ldots, 2 N-1 .\end{cases}
$$

Consequently, the coefficient vector $\left(c_{0}, \ldots, c_{2 N-1}\right)^{T}$ dose not depend on $t$ such that we only need to solve the linear system (56) once for every $t$.

Using a similar way, we can give a class of generalized modulating functions satisfying the properties $\left(P_{1}\right),\left(P_{2}\right),\left(P_{5}\right)$ and $\left(P_{6}\right)$ required in Corollary 2.

Corollary 3 Let $[a, t] \subset \mathbb{R}, n \in \mathbb{N}, N \in \mathbb{N}^{*}$ with $n \leq N-1$, and $f_{n}$ be a polynomial with the following form:

$$
f_{n}(\tau)=\sum_{i=0}^{2 N-1} d_{i}(\tau-a)^{N+i},
$$

where the coefficient vector $\left(d_{0}, \ldots, d_{2 N-1}\right)^{T}$ is the solution of the following system:

$$
B_{n}\left(\begin{array}{c}
d_{0} \\
\vdots \\
d_{2 N-1}
\end{array}\right)=I_{n}
$$

where for $j=0, \ldots, 2 N-1, I_{n}(n)=1, I_{n}(j)=0$ with $j \neq n$, and for $i=0, \ldots, 2 N-1$,

$$
B_{n}(j, i)= \begin{cases}\frac{\sum_{k=0}^{N-1-j}(-1)^{k} a_{j+1+k} \frac{(N+i) !}{(N+i-k) !}(t-a)^{N+i-k},}{(1}(t-a)^{2 N+i+\beta-j}, & \text { for } j=0, \ldots, N-1, \\ \frac{1}{2 N+i+\beta-j}(\text { for } j=N, \ldots, 2 N-1 .\end{cases}
$$

Then, $f_{n}$ is a generalized modulating function satisfying the properties $\left(P_{1}\right),\left(P_{2}\right),\left(P_{5}\right)$ and $\left(P_{6}\right)$ on $[a, t]$.

\section{References}

[1] B. Ross, The development of fractional calculus 1695-1900, Hist. Math., 4, 1, pp. 75-89, 1977.

[2] K.B. Oldham and J. Spanier, The Fractional Calculus, Academic Press, New York 1974. 
[3] I. Podlubny, Fractional Differential Equations, Academic Press, New York, NY, USA, 1999.

[4] A.A. Kilbas, H.M. Srivastava and J.J. Trujillo, Theory and Applications of Fractional Differential Equations, vol. 204 of North-Holland Mathematics Studies, Elsevier, Amsterdam, The Netherlands, 2006.

[5] A. Oustaloup, B. Mathieu and P. Lanusse, The CRONE control of resonant plants: application to a flexible transmission, European Journal of Control, vol. 1, no. 2, pp. 113-121, 1995.

[6] A. Oustaloup, J. Sabatier and X. Moreau, From fractal robustness to the CRONE approach, in Proc. ESAIM: Proceedings, pp. 177-192, Dec. 1998.

[7] A. Oustaloup, J. Sabatier and P. Lanusse, From fractal robustness to the CRONE control, FCAA, vol. 1, no. 2, pp. 1-30, Jan. 1999.

[8] V. Pommier, J. Sabatier, P. Lanusse and A. Oustaloup, CRONE control of a nonlinear hydraulic actuator, Control Engineering Practice, vol. 10, pp. 391-402, Jan. 2002.

[9] B. Mathieu, P. Melchior, A. Oustaloup, and Ch. Ceyral, Fractional differentiation for edge detection, Signal Processing, vol. 83, pp. 2421-2432, 2003.

[10] B. Guo, J. Li, and H. Zmuda, A new FDTD formulation for wave propagation in biological media with colecole model, IEEE Microwave and Wireless Components Letters, vol. 16, no. 12, pp. 633-635, 2006.

[11] M. Benmalek, and A. Charef, Digital fractional order operators for R-wave detection in electrocardiogram signal, IET Signal Processing, vol. 3, no. 5, pp. 381-391, 2009.

[12] R. Magin, M.D. Ortigueira, I. Podlubny and J. Trujillo, On the fractional signals and systems, Signal Processing, vol. 91, pp. 350-371, 2011.

[13] J.A.T. Machado, Calculation of fractional derivatives of noisy data with genetic algorithms, Nonlinear Dynamics, vol. 57, pp. 253-260, 2009.

[14] J.A.T. Machado, Exploiting sensor redundancy for the calculation of fractional derivatives in the presence of noise, Signal Processing, vol. 92, pp. 204-209, 2012.

[15] D.L. Chen, Y.Q. Chen and D.Y. Xue, Digital Fractional Order Savitzky-Golay Differentiator, IEEE Transactions on Circuits and Systems II: Express Briefs, vol. 58, no. 11, pp. 758-762, 2011.

[16] D.Y. Liu, O. Gibaru, W. Perruquetti and T.M. Laleg-Kirati, Fractional order differentiation by integration with Jacobi polynomials, in Proc. 51st IEEE Conference on Decision and Control, Hawaii, USA, 2012. 
[17] D.Y. Liu, T.M. Laleg-Kirati, O. Gibaru and W. Perruquetti, Fractional Order Numerical Differentiation with B-Spline Functions, the International Conference on Fractional Signals and Systems, Gent, Belgium, 2013.

[18] A. Savitzky and M.J.E. Golay, Smoothing and differentiation of data by simplified least squares procedures, Anal. Chem., vol. 36, no. 8, pp. 1627-1639, 1964.

[19] D.Y. Liu, O. Gibaru and W. Perruquetti, Differentiation by integration with Jacobi polynomials, J. Comput. Appl. Math., vol. 235, no. 9, pp. 3015-3032, 2011.

[20] S. Ibrir and S. Diop, A numerical procedure for filtering and efficient high-order signal differentiation, Internat. J. Appl. Math. Comput. Sci., vol. 14, pp. 201-208, 2004.

[21] M. Fliess and H. Sira-Ramírez, Reconstructeurs d'états, C.R. Acad. Sci. Paris, Ser. I, vol. 338, pp. 91-96, 2004.

[22] Y. Tian, T. Floquet and W. Perruquetti, Fast state estimation in linear time-varying systems: an algebraic approach, in Proc. 47th IEEE Conference on Decision and Control, Cancun Mexique, 2008.

[23] Y. Tian, T. Floquet and W. Perruquetti, Fast state estimation in linear time-invariant systems: an algebraic approach, in 16th Mediterranean Conference on Control and Automation (MED'08), Ajaccio, Corsica, France, 2008.

[24] M. Fliess, Analyse non standard du bruit, C.R. Acad. Sci. Paris, Ser. I, vol. 342, pp. 797-802, 2006.

[25] M. Fliess, Critique du rapport signal à bruit en communications numériques - Questioning the signal to noise ratio in digital communications, International Conference in Honor of Claude Lobry, Revue africaine d'informatique et de Mathématiques appliquées, vol. 9, pp. 419-429, 2008.

[26] M. Fliess and H. Sira-Ramírez, An algebraic framework for linear identification, ESAIM Control Optim. Calc. Variat., vol. 9, pp. 151-168, 2003.

[27] M. Mboup, C. Join and M. Fliess, Numerical differentiation with annihilators in noisy environment, Numerical Algorithms, vol. 50, no. 4, pp. 439-467, 2009.

[28] M. Mboup, C. Join and M. Fliess, A revised look at numerical differentiation with an application to nonlinear feedback control, in Proc. 15th Mediterranean conference on Control and automation (MED'07), Athenes, Greece, 2007.

[29] D.Y. Liu, O. Gibaru and W. Perruquetti, Error analysis of Jacobi derivative estimators for noisy signals, Numerical Algorithms, vol. 58, no. 1, pp. 53-83, 2011. 
[30] D.Y. Liu, O. Gibaru and W. Perruquetti, Convergence Rate of the Causal Jacobi Derivative Estimator, Curves and Surfaces 2011, LNCS 6920 proceedings, pp. 45-55, 2011.

[31] D.Y. Liu, O. Gibaru and W. Perruquetti, Synthesis on a class of algebraic differentiators and application to nonlinear observation, the 33rd Chinese Control Conference, Nanjing, China, 2014.

[32] J.R. Trapero, H. Sira-Ramírez and V.F. Battle, An algebraic frequency estimator for a biased and noisy sinusoidal signal, Signal Processing, vol. 87, pp. 1188-1201, 2007.

[33] M. Mboup, Parameter estimation for signals described by differential equations, Applicable Analysis, vol. 88, pp. 29-52, 2009.

[34] R. Ushirobira, W. Perruquetti, M. Mboup and M. Fliess, Algebraic parameter estimation of a multi-sinusoidal waveform signal from noisy data, in Proc. 2013 European Control Conference, Zurich, Switzerland, 2013.

[35] W. Perruquetti, P. Fraisse, M. Mboup and R. Ushirobira, An algebraic approach for Humane posture estimation in the sagital plane using accelerometer noisy signal, in Proc. 51st IEEE Conference on Decision and Control, Hawaii, USA, 2012.

[36] D.Y. Liu, O. Gibaru and W. Perruquetti, Parameters estimation of a noisy sinusoidal signal with time-varying amplitude, in Proc. 19th Mediterranean conference on Control and automation (MED'11), Corfu, Greece, 2011.

[37] D.Y. Liu, O. Gibaru, W. Perruquetti, M. Fliess and M. Mboup, An error analysis in the algebraic estimation of a noisy sinusoidal signal, in Proc. 16th Mediterranean conference on Control and automation (MED'08), Ajaccio, France, 2008.

[38] D.Y. Liu, O. Gibaru and W. Perruquetti, Non-asymptotic fractional order differentiators via an algebraic parametric method, 1st International Conference on Systems and Computer Science, Villeneuve d'ascq, France, 2012.

[39] N. Gehring and J. Rudolph, An Algebraic Approach for Identification of Linear Systems with Fractional Derivatives, arXiv:1302.4071, 2013.

[40] M. Shinbrot, On the analysis of linear and nonlinear dynamic systems from transient-response data, National Advisory Committee for Aeronautics NACA, Technical Note 3288, Washington, 1954.

[41] H.A. Preising and D.W.T. Rippin, Theory and application of the modulating function method. I: Review and theory of the method and theory of the splinetype modulating functions, Computers and Chemical Engineering, vol. 17, pp. 1-16, 1993. 
[42] T.B. Co and S. Ungarala, Batch scheme recursive parameter estimation of continuous-time system using the modulating functions method, Automatica, vol. 33, no. 6, pp. 1185-1191, 1997.

[43] H. Unbehauen and G.P. Rao, A review of identification in continous-time systems, Annual Reviews in Control, vol. 222, pp. 145-171, 1998.

[44] G. Fedele and L. Coluccio, A recursive scheme for frequency estimation using the modulating functions method, Appl. Math. Comput., vol. 216, pp. 1393-1400, 2010.

[45] D.Y. Liu, T.M. Laleg-Kirati, O. Gibaru and W. Perruquetti, Identification of fractional order systems using modulating functions method, in Proc. 2013 American Control Conference, Washington, DC, USA, 2013.

[46] D.Y. Liu, T.M. Laleg-Kirati, W. Perruquetti and O. Gibaru, Non-asymptotic state estimation for a class of linear time-varying systems with unknown inputs, the 19th World Congress of the International Federation of Automatic Control, Cape Town, South Africa, 2014.

[47] M. Abramowitz and I.A. Stegun, editeurs, Handbook of mathematical functions, GPO, 1965.

[48] I. Podlubny and Y.Q. Chen, Adjoint fractional differential expressions and operators, in Proc. the ASME 2007 International Design Engineering Technical Conferences $\&$ Computers and Information in Engineering Conference IDETC/CIE 2007, Las Vegas, NV, September 4-7, 2007.

[49] A. Ralston, A first course in numerical analysis, McGraw-Hill, New York, 1965.

[50] K.S. Miller and B. Ross, An Introduction to the Fractional Calculus and Fractional Differential Equations, Wiley, New York, 1993. 


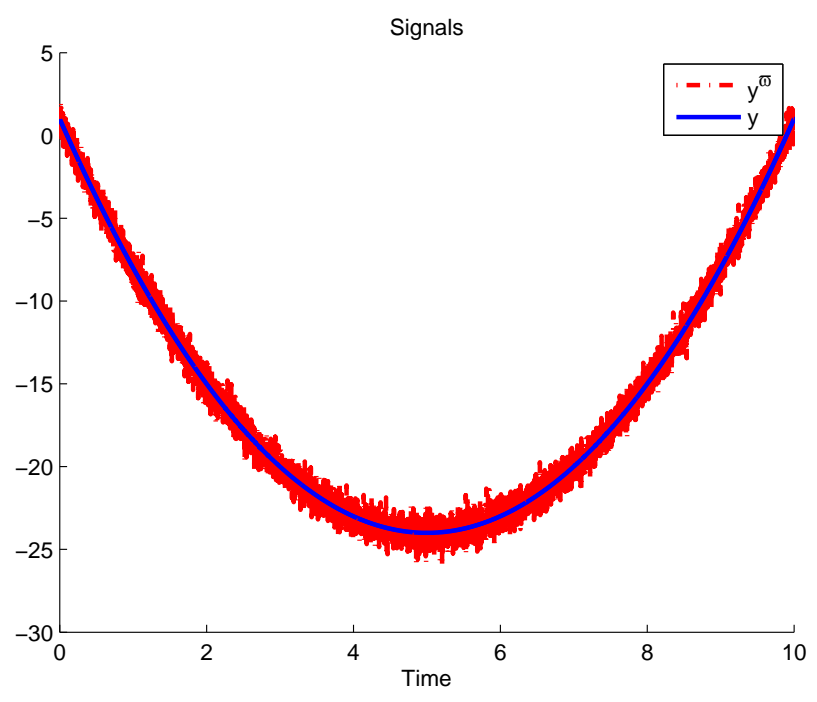

Figure 1: Example 1: Discrete polynomial signal $y\left(t_{i}\right)=t_{i}^{2}-10 t_{i}+1$ with $T_{s}=10^{-3}$, and its noisy observation corrupted by a zero-mean white Gaussian noise with $\mathrm{SNR}=30 \mathrm{~dB}$. 


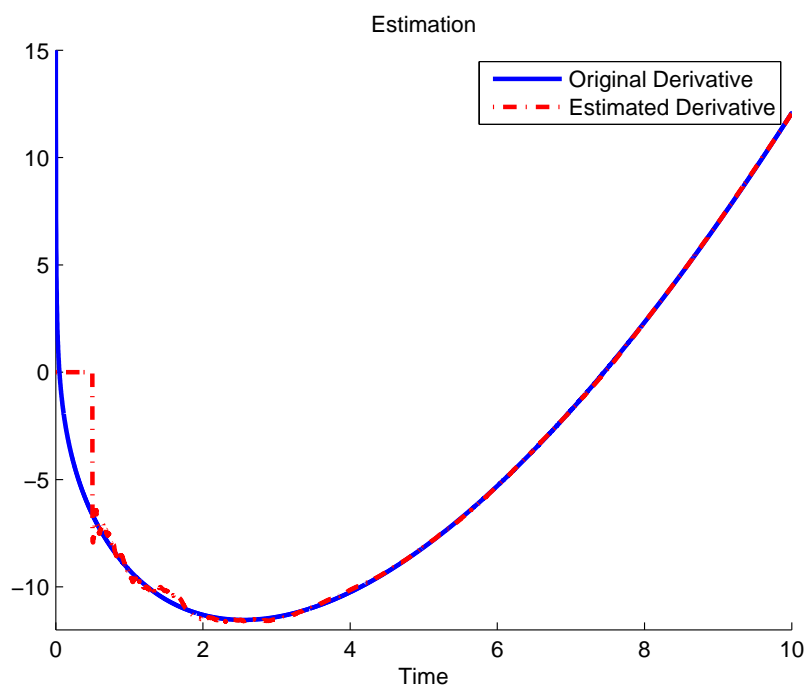

(a) Estimation of the $0.5^{\text {th }}$ order derivative of $y$.

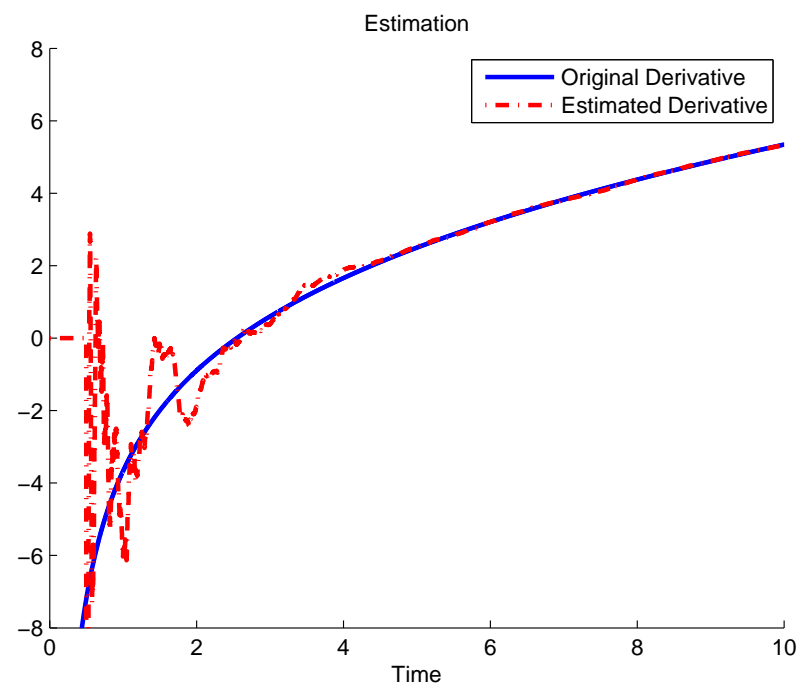

(b) Estimation of the $1.5^{\text {th }}$ order derivative of $y$.

Figure 2: Estimations of the $0.5^{t h}$ order and $1.5^{t h}$ order Riemann-Liouville fractional derivatives of the polynomial signal $y$, obtained by (40) and (41) respectively in the discrete noisy case. 


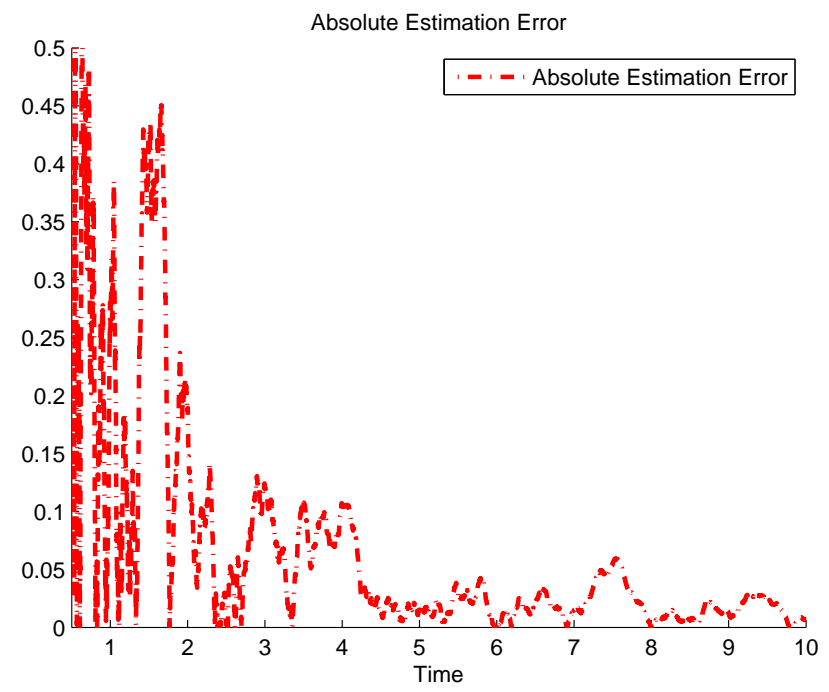

(a) Absolute estimation error for the estimation of the $0.5^{t h}$ order derivetine of $y$.

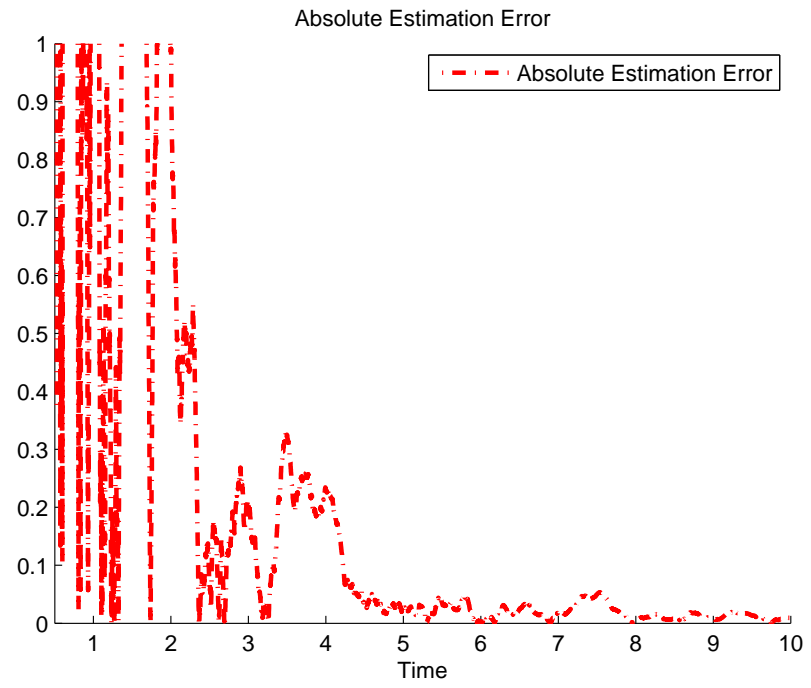

(b) Absolute estimation error for the estimation of the $1.5^{\text {th }}$ order derivetine of $y$.

Figure 3: Absolute estimation errors for the estimations obtained by (40) and (41) in the discrete noisy case.

24 


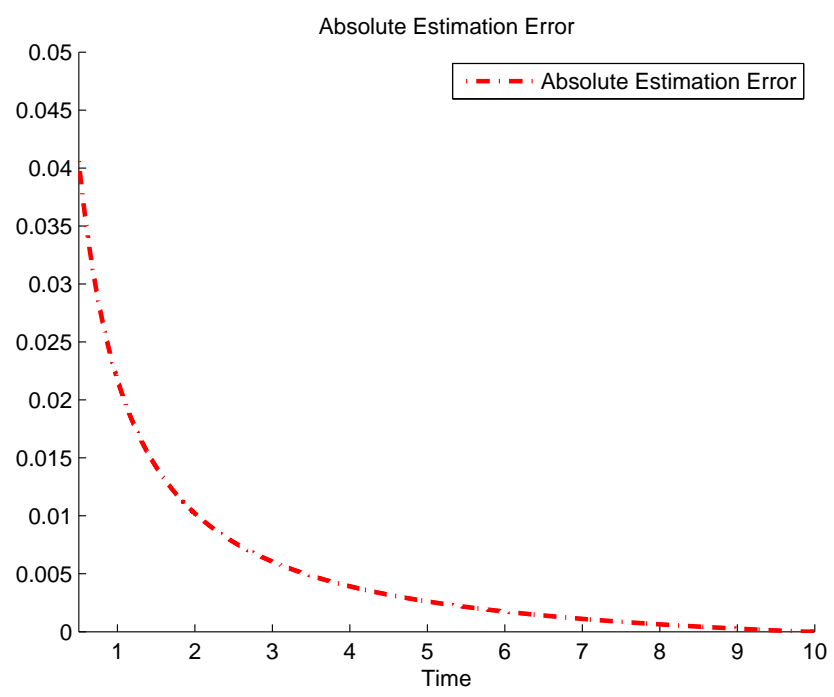

(a) Absolute estimation error for the estimation of the $0.5^{t h}$ order derivative of $y$.

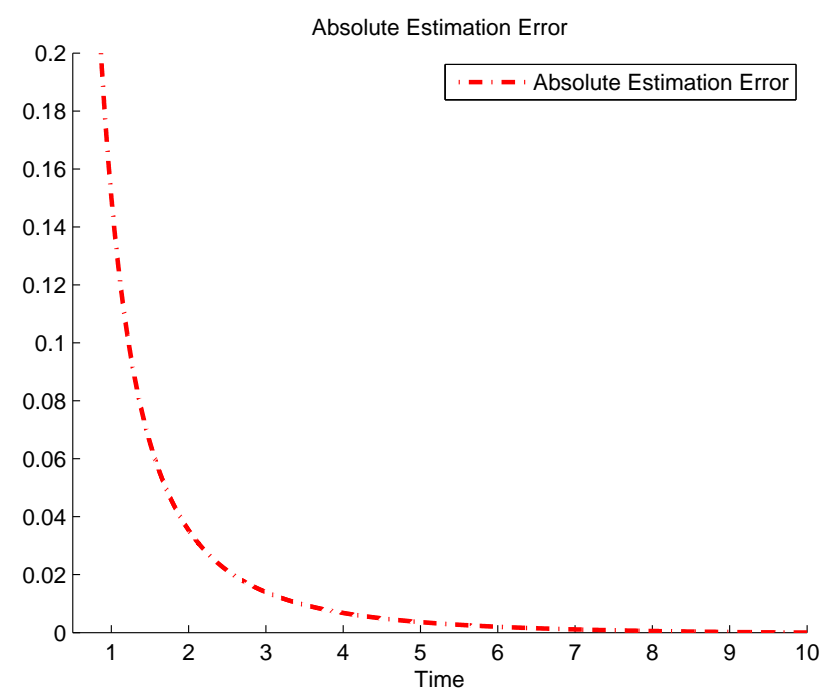

(b) Absolute estimation error for the estimation of the $1.5^{\text {th }}$ order derivative of $y$.

Figure 4: Absolute estimation errors for the estimations obtained by (40) and (41) in discrete noise fee case (numerical errors). 


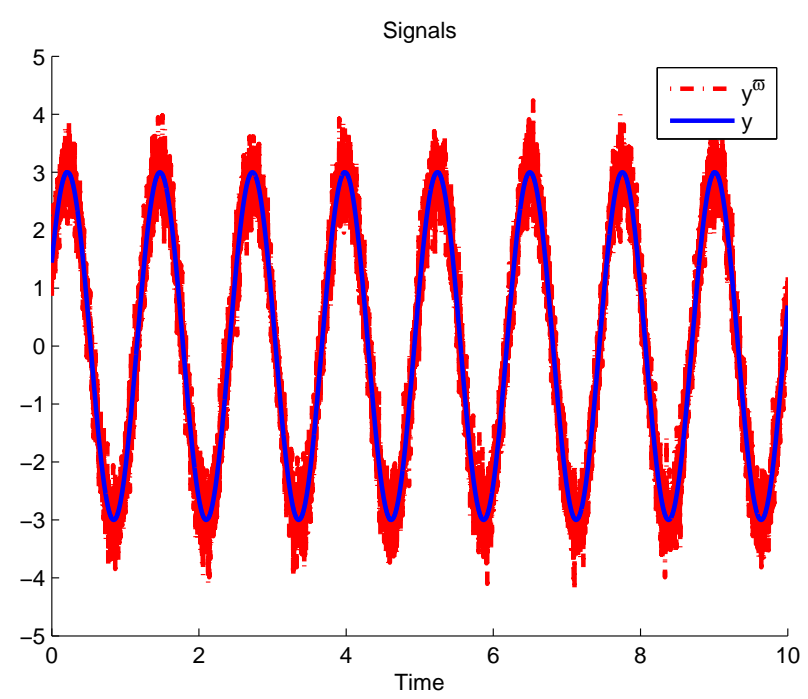

(a) Discrete sinusoidal signal $y$ and its noisy observation.

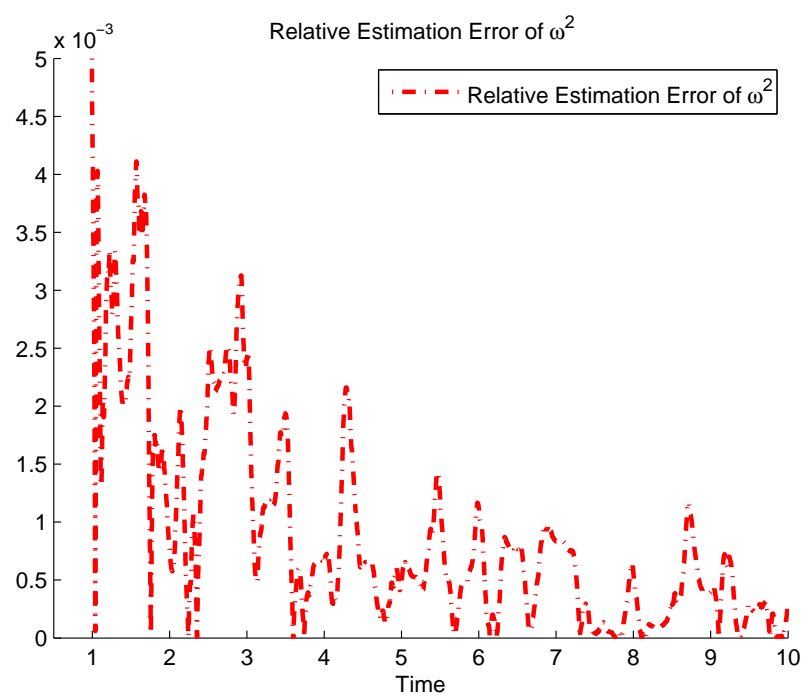

(b) Relative estimation error of $\omega^{2}$ obtained by (51) in the discrete noisy case.

Figure 5: Example 2: Discrete sinusoidal signal $y\left(t_{i}\right)=3 \sin \left(5 t_{i}+0.5\right)$ with $T_{s}=10^{-3}$, and its noisy observation corrupted by a zero-mean white Gaussian noise with $\mathrm{SNR}=15 \mathrm{~dB}$. 


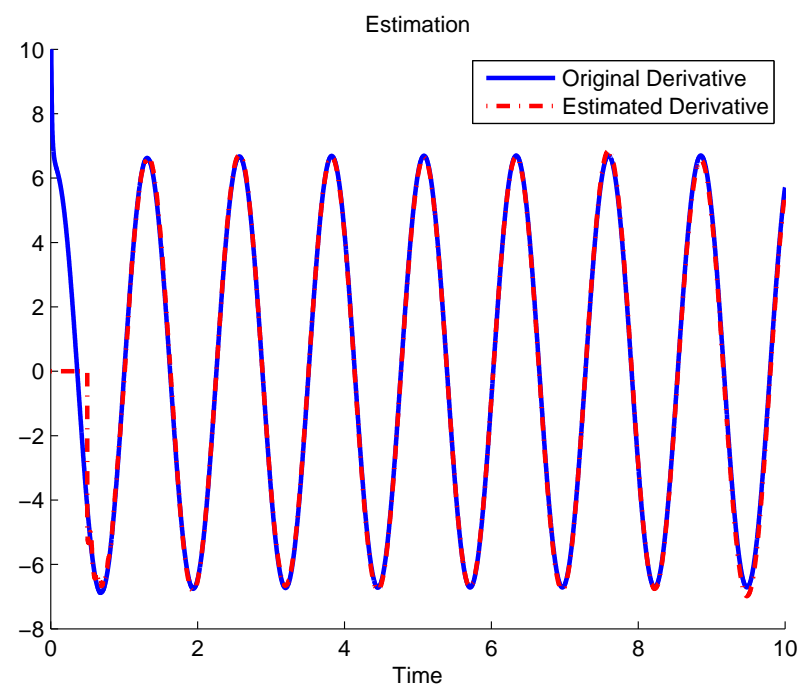

(a) Estimation of the $0.5^{\text {th }}$ order derivative of $y$.

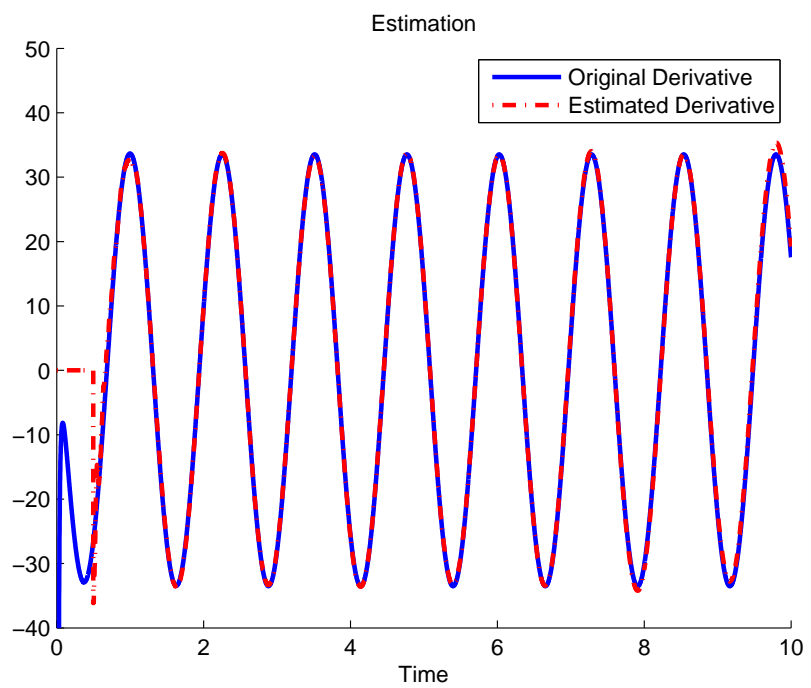

(b) Estimation of the $1.5^{\text {th }}$ order derivative of $y$.

Figure 6: Estimations of the $0.5^{t h}$ order and $1.5^{\text {th }}$ order Riemann-Liouville fractional derivatives of the sinusoidal signal $y$, obtained by (53) and (54) respectively in the discrete noisy case. 


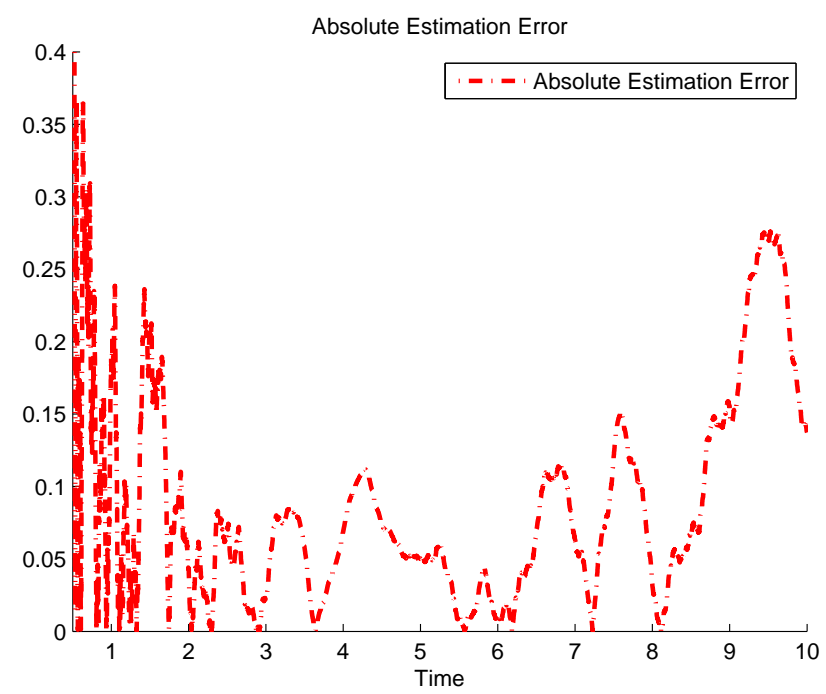

(a) Absolute estimation error for the estimation of the $0.5^{t h}$ order derivative of $y$.

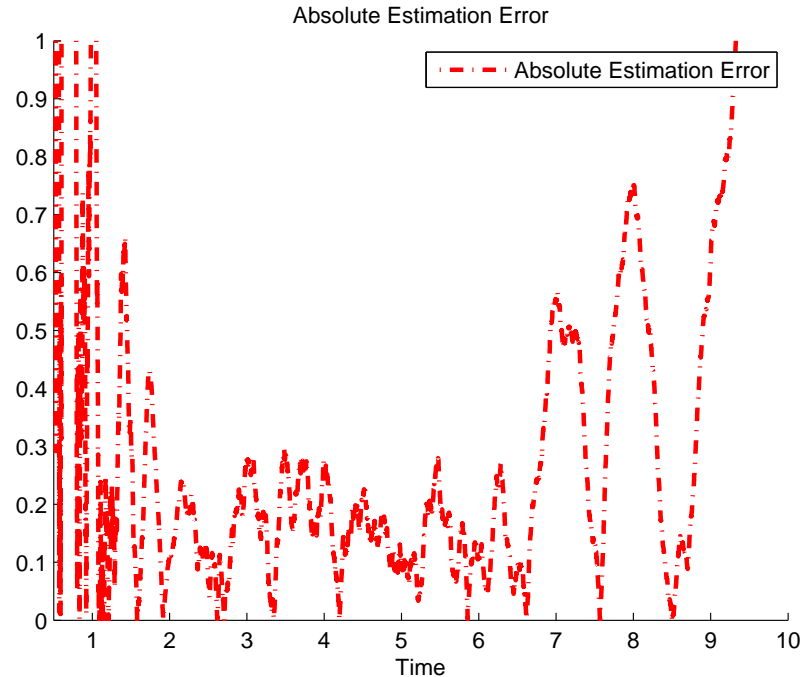

(b) Absolute estimation error for the estimation of the $1.5^{\text {th }}$ order derivative of $y$.

Figure 7: Absolute estimation errors for the estimations obtained by (53) and (54) in the discrete noisy case. 


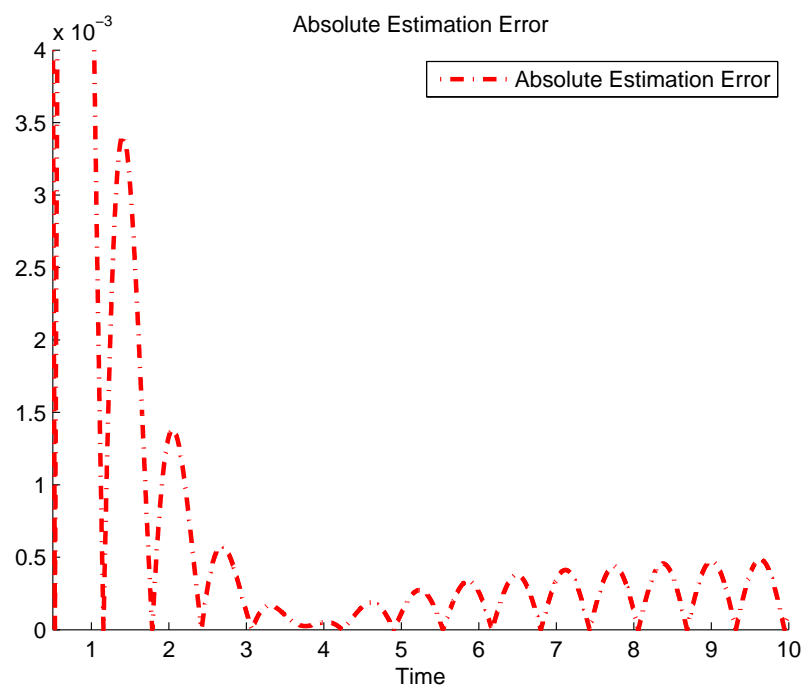

(a) Absolute estimation error for the estimation of the $0.5^{t h}$ order derivative of $y$.

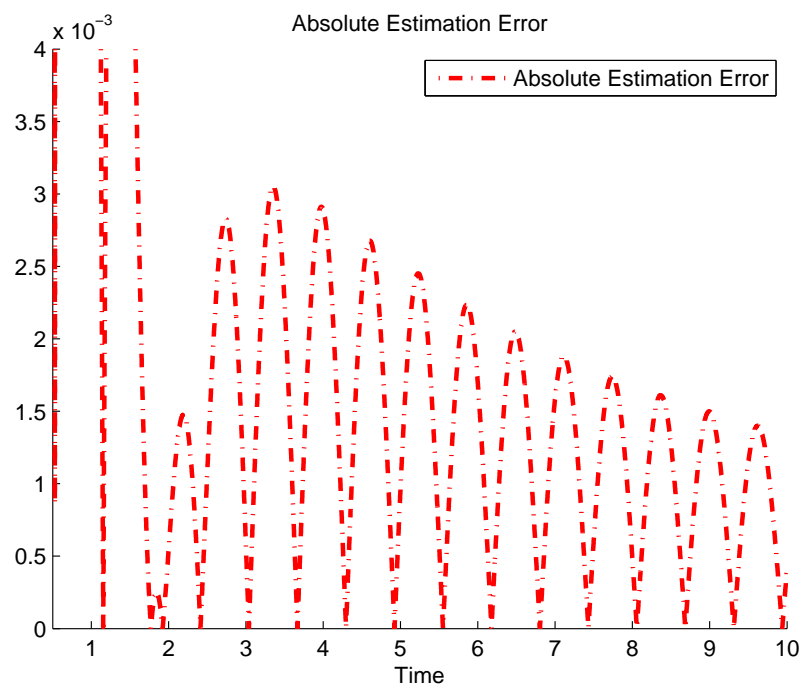

(b) Absolute estimation error for the estimation of the $1.5^{\text {th }}$ order derivative of $y$.

Figure 8: Absolute estimation errors for the estimations obtained by (53) and (54) in discrete noise fee case (numerical errors). 